\title{
Bioinformatics approach for prediction of metabolic capabilities for synthesis of essential vitamins and amino acids in human gut microbiome
}

\author{
G.A. Ashniev*, A.A. Arzamasov, S.N. Iablokov, D.A. Rodionov \\ Institute for Information Transmission Problems RAS, Moscow, Russia \\ *e-mail: escobar.morente@gmail.com
}

Key words: amino acids, vitamins, metagenomics, human, gut, bacteria, biosynthesis, metabolomics

Introduction: Bacteria are abundantly presented in almost every part of the world, living in sundry communities with complex interactions and various phenotype features. As soon as cooperation and rivalry within microbial communities is well known as central part of their stability, continuity and longevity, there is lack of knowledge about the general principles of vitamins amino acids metabolism, particularly biosynthesis. Understanding of basic features of amino acid formation is key for inner-community regulation and its influence on host organism.

Results: We used the metabolic subsystem approach implemented in SEED genomic platform to reconstruct essential biosynthetic pathways in bacteria that inhabit human gastrointestinal tract. We analyzed metabolic pathways for synthesis of 9 vitamins and 20 amino acids in 2228 human gut bacteria with sequenced genomes and inferred their vitamin and amino acid phenotypes (prototrophy or auxotrophy). We also analyzed genomic distributions of known uptake transporters for amino acids and vitamins. The predicted pathways allowed us to classify the studied organisms with respect in both to their biosynthetic and transport capabilities. The obtained metabolic phenotypes in reference genomes were applied to human stool samples from different published metagenomics. A unique approach for taxonomy-based mapping allowed us to compute the cumulative amino acid and vitamin biosynthetic phenotypes of microbial samples from a large number of metagenomics studies.

Conclusion: The studied bacteria showed high level of conservation of amino acid and vitamin biosynthesis phenotypes on the taxonomic level of species. Incomplete biosynthesis pathways for some intermediates suggest certain deficiencies could be alternatively supplemented by their metabolic precursors. Vitamin auxotrophic phenotypes are much more prevalent in the human gut microbiota, whereas the larger number of studied bacteria is capable of de novo synthesis of all 20 amino acids. Histidine and tryptophan have the most variable phenotypes. The comparison of metabolic profiles of gut microbiota between various population groups revealed applicability of this approach to classify microbiota and predict the functional consequences of dietary changes in composition of gut microbiota.

Acknowledgments: This research was supported by the Russian Science Foundation (grant No. 14-14-00289). 